\title{
V-BELT WINDING ALONG ARCHIMEDEAN SPIRALS DURING THE VARIATOR SPEED RATIO SHIFT
}

\author{
Francesco Sorge \\ Università di Palermo, Dipartimento di Meccanica \\ Viale delle Scienze, 90128 - Palermo, Italy \\ e-mail: sorge@dima.unipa.it
}

\begin{abstract}
Starting from a previous model for the shift mechanics of rubber belt variators, this lecture elaborates practical design formulas for the torque and the axial thrust making use of the very close resemblance of the belt path to a linear spiral of Archimedes along a large part of the arc of contact. In addition, as an alternative to the modern calculus tools, it is shown how the drive variables can be equally calculated applying some propositions of Archimedes' classical treatise $\pi \varepsilon \rho i$ ' $\varepsilon \lambda \imath \kappa \omega \nu$ (On Spirals).
\end{abstract}

\section{INTRODUCTION}

The usual operation of the continuously variable transmissions (CVT) for vehicles or motorcycles consists in a random continuous change of the speed ratio, where a gross radial motion of the belt toward the inside or outside of the groove is superimposed to the circular motion. Only a few approaches to the CVT transient mechanics can be found in the literature (see references in [1,2]) and a practical formulary is still missing. The present paper resumes the theory of [1] and constructs useful design formulas for the torque and the axial thrust. The full equation system is strongly non linear and its exact solution requires complex numerical procedures. Attempts at approximate solutions were carried out for some applicative cases [2], achieving a very fine agreement with experiments. Here, a much simpler formulation will be developed, taking advantage of the Archimedean spiral shape of the instantaneous belt line.

The involvement of Archimedes in the mechanics of machines was quite relevant and several ingenious devices are to be ascribed to him, both for the civil and military application, though such inventions arose more from practical occasional requirements than from his intimate disposition to this kind of activity [3,4]. Among many other interests, he was also concerned with the cable and belt mechanics and for example, his compound-pulley tackles ( $\pi 0 \lambda v \sigma \pi \alpha \sigma \tau \alpha)$ for the launch of very big ships are recorded by the historians. Nevertheless, he preferred the speculative aspects of the theoretical mechanics and the investigation on several mathematical and geometrical problems regarding plane and solid figures [3-7]. His treatise on spirals, though characterized by a limited divulgation 
in the past, may be recognized of a great modernity after more than two thousand years and may still yield alternative methods for the solution of today's mechanical problems, as will be shown also in the following.

The history of belt mechanics is traced by Gerbert in ref. [8]. Despite the extensive use of cable and rope devices in the antiquity, the theoretical analysis of the belt drives originates only in recent centuries, starting from the well known capstan formula of Euler-Eytelwein and proceeding with the fundamental distinction made by Grashof between the adhesive arc (Ruhebogen) and the sliding arc (Gleitbogen). The V-shaped belts were introduced by John Gates at the beginning of the $20^{\text {th }}$ century. Their analysis and their use in variable speed drives date from more recent times (Lutz, Worley, Dittrich, Gerbert) and also the author of the present paper has been working in this research area during the last years.

\section{BELT-PULLEY COUPLING}

A scheme of the belt element with the wall forces is represented in Fig. 1a, while Fig. $1 \mathrm{~b}$ shows some details about the geometry and kinematics of the belt path. These figures may be used as a reference for the notation.

Putting $\theta=\theta(t)$ and $r=r[t, \theta(t)]$ along the trajectory of a belt element, one has $d r / d t=\dot{r}+\dot{\theta} r^{\prime}$, where dots and primes indicate the differentiation with respect to $t$ and $\theta$ respectively. Moreover, letting $x=\left(r_{\infty}-r\right) / r_{\infty}$ be the dimensionless elastic penetration of the belt, where $r_{\infty}$ is the nominal radius for infinite transverse stiffness of the belt, the self-evident geometrical relationship $r^{\prime}=-r \tan \chi$ gives

$$
x^{\prime}=(1-x) \tan \chi
$$

The above formulas give rise to the relationship $v \sin \delta=v \cos \delta \tan \chi-\dot{r}$, while the triangle of velocities points out that $v \cos \delta-\omega r-v \sin \delta \tan \gamma=0$, and such two relationships yield $v \cos \delta(1-\tan \chi \tan \gamma)=\omega r(1-\rho \tan \gamma)$, where $\rho=\dot{r} /(\omega \cdot r) \cong \dot{r}_{\infty} /\left(\omega \cdot r_{\infty}\right)$ is the dimensionless shift speed. Therefore, if $\rho=\tan \chi$, then $v \cos \delta=\omega r$ and $v \sin \delta=0$, i. e. there is adhesion between the belt and the pulley and one has $x^{\prime}=(1-x) \rho$ by Eq. (1), so that the belt has the shape of a logarithmic spiral. Nevertheless, as $x<1$, the instant path can be confused with a linear spiral of Archimedes.

Indicating the longitudinal elongation with $\varepsilon=T / S_{l}, T$ and $S_{l}$ being the belt force and the longitudinal stiffness, the usual order of magnitude of $x$, $\chi, \varepsilon$ and $\rho$ is of a few thousandths. Then, combining the Eulerian and Lagrangian formulations of the mass conservation condition with reference 


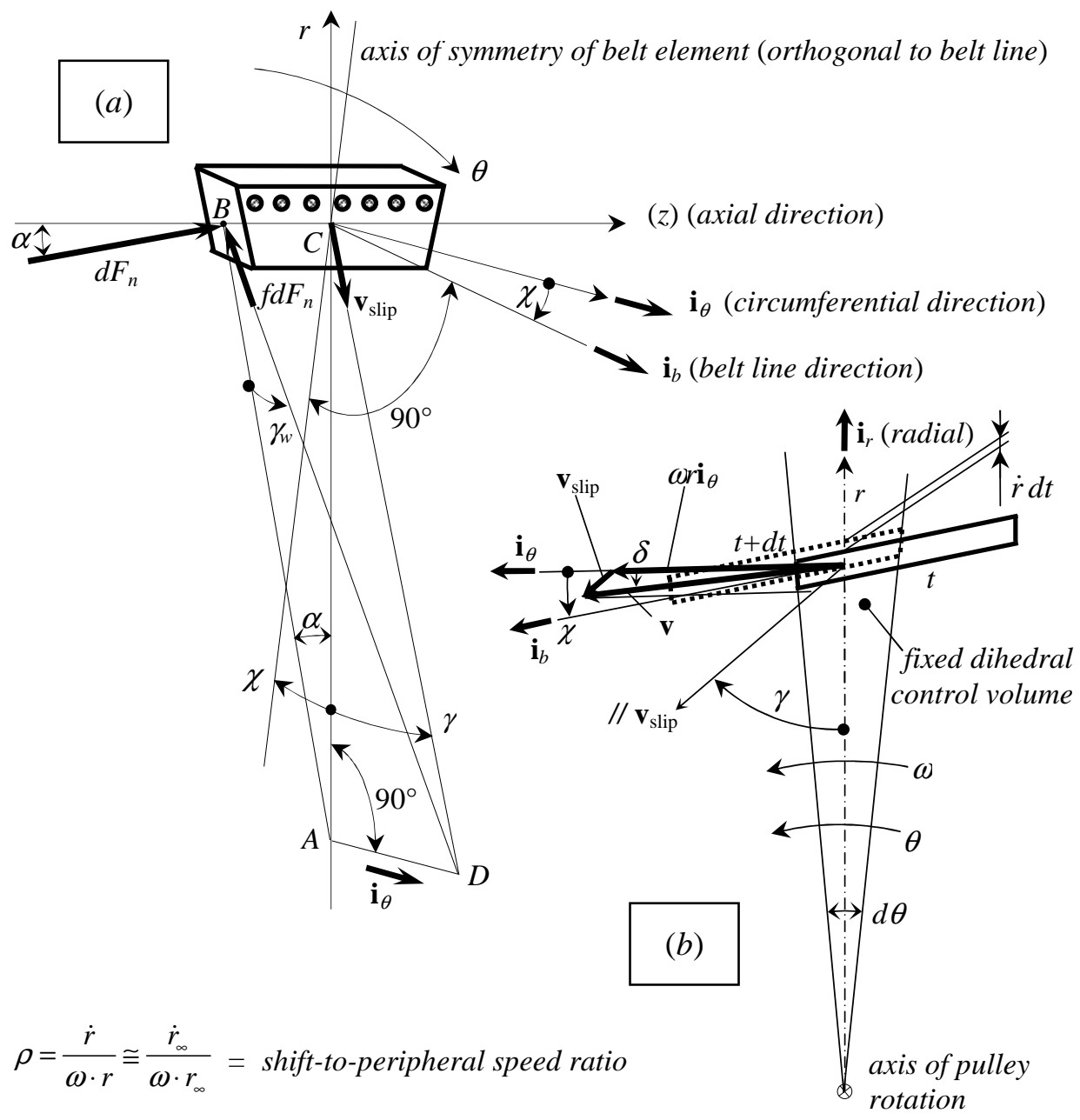

Fig.1a) Belt-pulley interaction. Tetrahedron of rotational (ACD), meridian $(A B C)$, sliding $(B C D)$ and wall-tangent $(A B D)$ planes.

Fig.1b) Control volume. Triangle of velocities.

\section{NOTATION}

$d F_{n}$ and $f d F_{n}=$ normal and frictional elementary wall forces

$\alpha=$ groove half-angle, $\delta=$ belt velocity angle, $\chi=$ belt penetration angle

$\gamma=$ sliding angle in plane of rotation, $\gamma_{w}=$ sliding angle on pulley wall $\left(\tan \gamma_{w}=\right.$ $\cos \alpha \tan \gamma)$

$r=$ belt radius, $\theta=$ angular coordinate

$\mathbf{v}=$ belt velocity, $\mathbf{v}_{\text {slip }}=$ slip velocity in rotation plane, $\rho=$ dimensionless shift speed, $\omega=$ pulley angular velocity 
to the dihedral control volume of Fig. $1 \mathrm{~b}$ and neglecting small terms, it is possible to arrive, as in [1], at the relationship

$$
u^{\prime}=(1+u)\left[\frac{\varepsilon^{\prime}}{1+\varepsilon}+\tan \chi\left(1-\chi^{\prime}\right)\right]-\rho
$$

where $u$ is the dimensionless slip velocity in the circumferential direction:

$$
u=\frac{v_{\text {slip }}}{\omega \cdot r} \sin \gamma=\left(\frac{\tan \chi-\rho}{1-\tan \gamma \tan \chi}\right) \tan \gamma \rightarrow \tan \gamma=\frac{u}{(1+u) \tan \chi-\rho}
$$

Neglecting small terms, equilibrium yields $\left(T_{d} \mathbf{i}_{b}\right)^{\prime}+\mathbf{F}_{w}^{\prime} \cong 0$, where $T_{d}=T-\mu v_{b}^{2}$ is a "dynamic" belt force, $\mu$ the belt mass per unit length, $\mu v_{b}{ }^{2}$ the momentum flux along the belt and $\mathbf{F}_{w}^{\prime}$ the resultant wall force per unit angle. Likewise, it is possible to define the "dynamic" elongation $\varepsilon_{d}=T_{d} / S_{l}$. Splitting the vectorial equilibrium equation in the directions tangential and normal to the belt, defining the transverse elastic modulus $E_{z}$ and the transverse stiffness parameter $S_{t}=2 \tan \alpha E_{z} h r_{\infty}{ }^{2} / w$ ( $h$ and $w$ : belt height and width), formulating a transverse constitutive equation, one gets as a whole

$$
\begin{gathered}
S_{l} d \varepsilon_{d}=2\left[\sin \alpha \sin \chi+f\left(\cos \gamma_{w} \cos \alpha \sin \chi+\sin \gamma_{w} \cos \chi\right)\right] d F_{n} \\
S_{l} \varepsilon_{d}\left(1+\chi^{\prime}\right) d \theta=2\left[\sin \alpha \cos \chi+f\left(\cos \gamma_{w} \cos \alpha \cos \chi-\sin \gamma_{w} \sin \chi\right)\right] d F_{n} \\
d F_{z}=\left(\cos \alpha-f \cos \gamma_{w} \sin \alpha\right) d F_{n}=S_{t} x(1-x) d \theta / \cos \chi
\end{gathered}
$$

Eliminating $d F_{n}$ from Eqs. (4-5) and introducing the belt elastic parameter $k=2 \tan \alpha S_{t} / S_{l}$, the momentum balance leads to

$$
\begin{gathered}
\mathcal{E}_{d}^{\prime}=\frac{k x(1-x)(\tan \beta+\tan \chi)}{1-\frac{\tan \beta}{\cos ^{2} \alpha \tan \gamma}} \\
\chi^{\prime}=\frac{k x(1-x)(1-\tan \beta \tan \chi)}{\varepsilon_{d}\left(1-\frac{\tan \beta}{\cos ^{2} \alpha \tan \gamma}\right)}-1
\end{gathered}
$$

where $\tan \beta=f \sin \gamma /\left(f \cos \gamma+\sin \alpha \sqrt{1+\tan ^{2} \alpha \cos ^{2} \gamma}\right)$.

We have thus collected four $1^{\text {st }}$ order differential equations, (1), (2), (7), (8), and one parametric equation, ( $3 \mathrm{a}$ or b), in the five variables $x, \varepsilon_{d}$, 
$\chi, u$ and $\gamma$, where the first four are very small, while $\gamma$ may range between $-\pi$ and $+\pi$. This differential system is "degenerescent", as its order "degenerates" from four to three when neglecting all smaller terms, including $\chi^{\prime}$ in Eq. (8). The problem is then of the boundary layer type, so that a rather smooth variation of the variables is expected along most of the contact, but with sharp gradients near the boundaries, and the equations must be applied in their unabridged form to match all boundary conditions. The numerical solution shows a short "seating" region at the contact entrance, where the belt slides inward $(\gamma \cong 0)$, and a short "unseating" region at the exit, where it slides outward $(\gamma \cong \pm \pi)$. The belt force is nearly constant in both of them by Eq. (7), but the elastic penetration is subject to rapid changes, together with the belt curvature. Putting $\gamma \cong 0$ at the seating region exit, one may obtain $x_{i n} \cong \mathcal{E}_{d, \text { in }} / k_{1}$ by Eq. (8), where $k_{1}=k \tan (\alpha+$ $\arctan f) / \tan \alpha$ and this expression of $x_{\text {in }}$ gives a good approximation for the penetration at the start of the inner main region of contact.

ADHESIVE SUB-REGION. As proven in [1], a wide adhesive region where $\tan \chi=\rho$ must develop inside the arc of contact of the closing pulleys $(\rho>$ 0 ), both driver and driven, next to the seating region and bounded by the endpoints $U$ and $D$ (Upstream and Downstream). Here, all the previous relationships hold, except that $f$ must be replaced by a variable adhesion factor $f_{a} \leq f_{s}$, where $f_{s}$ is the coefficient of static friction, and $\gamma$ by the angle $\gamma_{a}$ of the resultant adhesion force in the plane of rotation. The adherence limit is reached when $f_{a}=f_{s}$. The adhesive conditions, $\tan \chi=\rho, u=0$, imply the constancy of the belt force, as $\mathcal{E}_{d}^{\prime}=0$ by (2), while Eq. (1) gives $x=\left(r_{\infty}-r\right) / r_{\infty}=1-\left(1-x_{U}\right) \exp \left[-\rho\left(\theta-\theta_{U}\right)\right]$, where $x_{U} \cong \varepsilon_{\text {in }} / k_{1}$,. Then, at a fixed time instant, the belt coils along a logarithmic spiral, which, since $x$ and $|\rho|$ are $\ll 1$, may be roughly confused with a linear spiral of Archimedes

$$
x \cong x_{U}+\rho\left(\theta-\theta_{U}\right) \rightarrow r \cong r_{U}-r_{\infty} \rho\left(\theta-\theta_{U}\right)
$$

This spiral develops very slightly inward in the motion direction as $\rho>0$ and $\rho<<1$, but the belt radius increases at each fixed angular position due to the pulley rotation.

The small variables $x, \varepsilon_{d}, \chi$ and $u$ are obviously continuous when entering/leaving the adhesion region, while $\gamma$ and $f$ are always discontinuous with $\gamma_{a}$ and $f_{a}$ at $D$, but are continuous at $U$ if $f_{a}(U)=f$.

ADHESIVE-LIKE SUB-REGION. No adhesive contact may develop in the opening pulleys, but the growth of sufficiently large regions of contact requires the presence of adhesive-like regions, where the adhesion condition $\tan \chi=\rho$ is just approached but not fulfilled. Here, the slip 
velocity $\mathbf{v}_{\text {slip }}$ and the sliding angle $\gamma$ are rather small, whence we get $\mathcal{\varepsilon}_{d}^{\prime} \cong \rho$ $-\tan \chi \cong$ constant and $\mathcal{E}_{d} \cong k_{1} x$ by Eqs. (2) and (8). Moreover, as the trend of $\chi$ appears rather flat inside the adhesive-like region, the second derivative $\chi^{\prime \prime}$ must tend to vanish as well. Then, differentiating Eq. (8) and retaining only the dominant terms, it is possible to get $\chi^{\prime \prime} \cong[\tan \chi-(\rho-$ $\left.\tan \chi) / k_{1}\right] / x \cong 0$, whence $\tan \chi \cong \rho /\left(1+k_{1}\right)$ and the approximate gradients $x^{\prime} \cong \rho /\left(1+k_{1}\right)$ and $\varepsilon_{d}^{\prime} \cong k_{1} \rho /\left(1+k_{1}\right)$ are obtainable. These results permit constructing approximate solutions for the adhesive-like sub-regions:

$$
\begin{gathered}
x=\frac{\varepsilon_{d, i n}}{k_{1}}+\frac{\rho}{1+k_{1}}\left(\theta-\theta_{\text {in }}\right) \rightarrow r=r_{\text {in }}-\frac{r_{\infty} \rho}{1+k_{1}}\left(\theta-\theta_{i n}\right) \\
\mathcal{E}_{d}=\varepsilon_{d, \text { in }}+\frac{k_{1} \rho}{1+k_{1}}\left(\theta-\theta_{\text {in }}\right)
\end{gathered}
$$

Summing up, spiral-shaped paths grow up in the closing and opening shift phases. In the former ones, the belt force keeps constant due to adhesion, while a slight tension variation occurs in the last ones due to a small creep motion. Moreover, it is noteworthy that the sum of the gradients of $x$ and $\varepsilon_{d}$ is roughly equal to $\rho$ in both the shift operations. Figure 2 shows two belt paths schematically for a shift up and a shift down phase respectively. The belt velocity vectors are such that the belt radius increases or decreases in the closing or opening pulley respectively.

\section{NUMERICAL RESULTS}

The shift model can be dealt with as an initial value problem, starting the integration from one of the contact endpoints, e. g. the exit point $E$, where of course the belt penetration $x_{E}$ must be zero, and moving backward until $x$ vanishes again and a complete solution has been achieved. A great care must be put in the control of the integration step, reducing its width on approaching the adhesive or adhesive-like regions to avoid numerical instability. An iterative procedure must be followed, correcting successively the starting values by a sort of shooting technique, until all the external boundary conditions are fulfilled, i. e. for the contact width $\Theta$ $=\theta_{\text {exit }}-\theta_{\text {entrance }}$, the applied torque $\left(\varepsilon_{d, \text { exit }}-\varepsilon_{d, \text { entrance }}\right) S_{l} r_{\infty}$ and the axial thrust $F_{z}=\int_{\text {wrap arc }} d F_{z}$. Moreover, in the case of a closing pulley, the backward integration proceeds until the condition $\tan \chi=\rho$ is attained at the downstream adhesion boundary $D$, continues along the adhesive arc, according to the previous adhesive model until the condition $f_{a}=f$ is attained, assuming equal coefficients of static and sliding friction, and goes on upstream in the seating region. In general :1) a decrease of the exit 

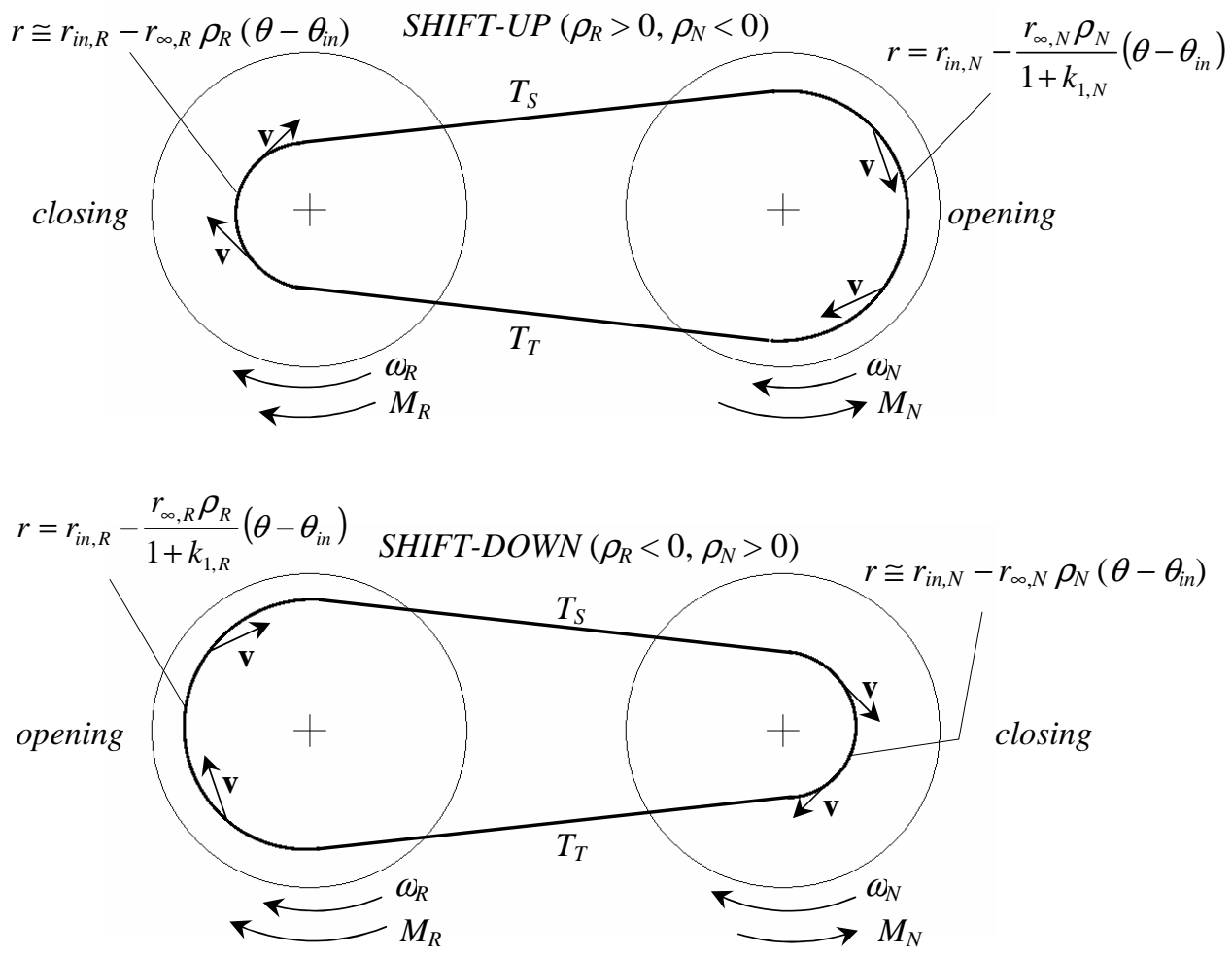

Fig. 2. Examples of shift-up and shift-down phases with magnified spiral shape of the belt path. $\left|\rho_{R}\right| \cong\left|\rho_{N}\right| \cong 0.05, k_{l, R} \cong k_{1, N} \cong 0.5$. Subscripts $R$ and $N$ : driveR and driveN pulleys.

angle $\chi_{E}$, which is always negative, produces an increase of the contact width; 2) a small increase of the sliding angle $\gamma_{E}$, which must be very close to $\pm \pi$, tends to change the pulley behaviour from driven to driver; 3 ) an increase of the belt elongation $\varepsilon_{d, E}$ produces an increase of the axial thrust.

Figures 3 to 6 show examples of numerical results for the four possible operative conditions of a pulley: driver/driven, opening/closing. The solutions were obtained fixing the shift-to-peripheral speed ratio $\rho$, the "centrifugal elongation" $\mu v_{b}{ }^{2} / S_{l}$ and the exit values of $\gamma_{E}$ and $\varepsilon_{d, E}=\left(T_{E}-\right.$ $\left.\mu v_{b}^{2}\right) / S_{l}$, where $T_{E}$ is the belt tension in the free strand downstream. The third initial value $\chi_{E}(<0)$ was corrected systematically to get a pre-fixed wrap width.

The ratio $f_{a} / f$ is also reported for the adhesive case (closing p.) and a 

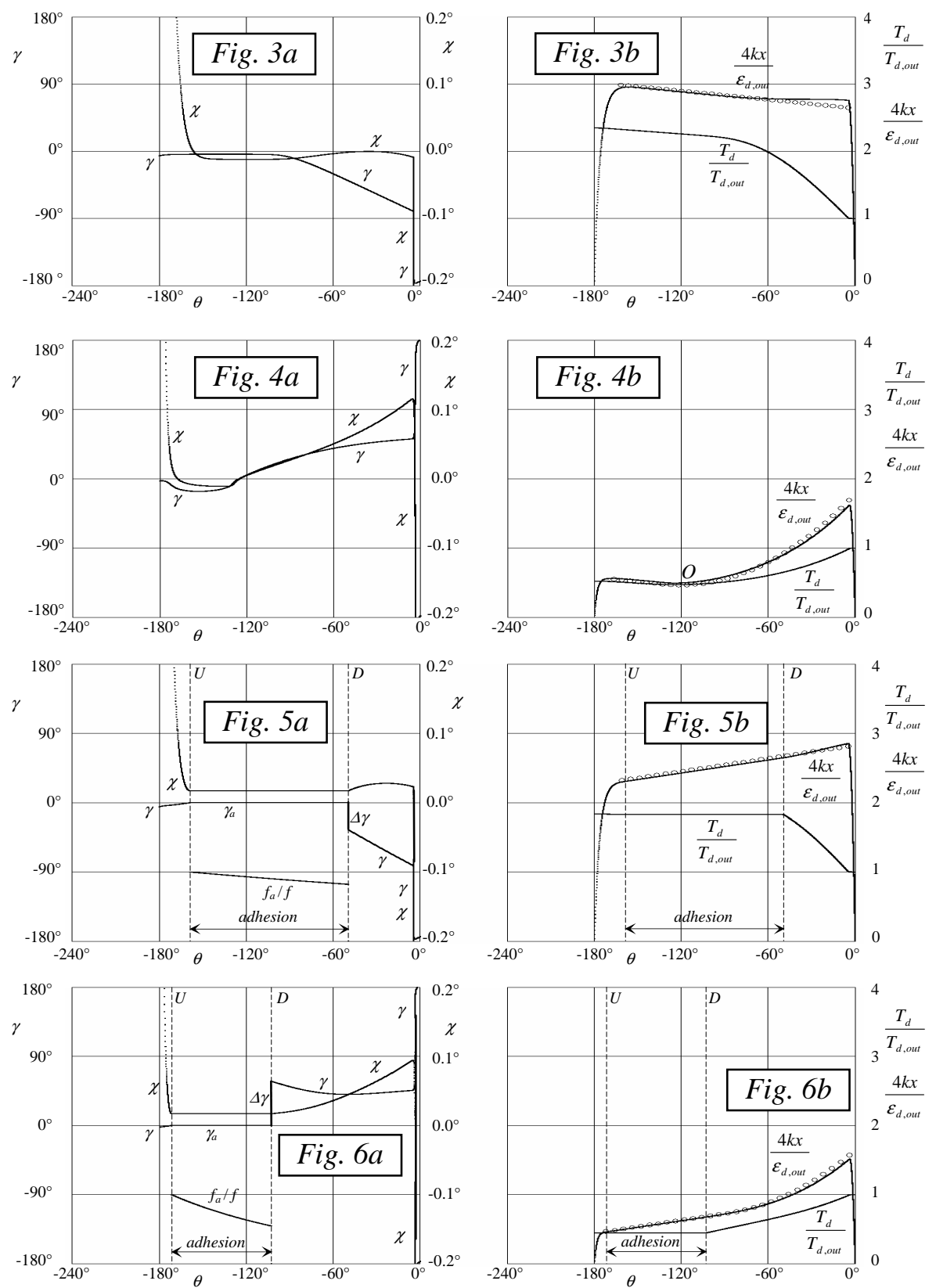

Figg.3-6. Solutions from unabridged equations (continuous line) and interpolating functions (dots)

$k=0.15, \alpha=13^{\circ}, f=0.4, \Theta=180^{\circ}, \rho=0.0003, \mu v_{b}=0.0001$.

3) opening driver $p$., $\gamma_{E}=186^{\circ}$; 4) opening driven $p$., $\gamma_{E}=182^{\circ}$;

5) closing driver p., $\gamma_{E}=186^{\circ}$; б) closing driven p., $\gamma_{E}=183^{\circ}$. 
jump $\Delta \gamma$ is observable at the downstream boundary, due to the jump from $f_{a}$ to $f$.

The belt angle $\chi$ is quite small everywhere, save in the seating and unseating regions, where it is affected by sharp negative gradients. Moreover, the sliding angle $\gamma$ is close to 0 and to $\pm \pi$ in these short boundary regions, where thus the belt tension is nearly constant. Nevertheless, a sharp variation of $\gamma$ occurs when passing from the main internal region of contact into the unseating region. Therefore, indicating with the subscripts $\ldots$ in and $\ldots$ out the ends of the wide inner region, the previous initial relationship $\mathcal{E}_{d, i n} \cong k_{1} x_{i n}$ is valid, but a similar relationship cannot be written at the end.

Observing the diagrams of the closing pulleys, the belt tension $T_{d}$ is constant in the adhesive sub-region, where the elastic penetration $x$ varies in practice linearly with $\theta$ according to Eq. (9). Likewise, linear trends of $T_{d}$ and $x$ may be observed in the adhesive-like regions of the opening pulleys, according to Eqs. (10a,b). This suggests approximate solutions.

\section{PRACTICAL FORMULARY}

Neglecting the short seating and unseating regions, the linear trend of $x$ in the adhesive or adhesive-like sub-regions may be conveniently used for the approximate integration of Eq. (6) for the axial thrust $F_{z}=\int_{\text {wrap arc }} d F_{z} \cong$ $\int_{\text {wrap arc }} S_{t} x d \theta$.

Considering the driver pulley and observing several solutions, this linear trend may be approximately prolonged inside the downstream main sliding region as far as its endpoint, both for the closing and opening phases. Since only the area under this plot is of interest and not the exact shape of the locus, this approximation may give an extraordinary tool for the practical calculation of the driver pulley performance:

$$
F_{z, R}=\frac{\left(T_{T}-\mu v_{b}^{2}\right) \Theta_{R}}{2 \tan (\alpha+\arctan f)}+\frac{\rho_{R} S_{t, R} \Theta_{R}^{2}}{2+k_{1, R}\left[1-\operatorname{sgn}\left(\rho_{R}\right)\right]}
$$

where the subscripts $\ldots R$ and $\ldots T$ refer to the drive $R$ behaviour and to the tight strand. Notice that the belt parameters vary during the shift phase and, in particular, $S_{t, R}$ and $k_{1 R}$ vary with the square of the radius.

As regards the driven pulleys, it is better to divide the region of contact in two sub-regions: a first adhesive or adhesive-like sub-region, where Eqs. (9) or (10a) hold, and a second main sliding sub-region, where the solutions may be constructed according to the following reasoning, based on the observation that the penetration-to-elongation ratio $x / \varepsilon_{d}$ and their 
differential ratio $d x / d \varepsilon_{d}$ tend roughly to the same "asymptotic" value on approaching the endpoint: $d x_{\text {out }} / d \varepsilon_{d, \text { out }} \rightarrow x_{\text {out }} / \mathcal{E}_{d, \text { out }} \rightarrow$ constant $=m$.

Neglecting $\chi, \chi^{\prime}$ and putting $1-x \cong 1$, Equations (7) and (8) change into $\varepsilon_{d}^{\prime} \cong \varepsilon_{d} \tan \beta \cong \cos ^{2} \alpha \tan \gamma\left(\varepsilon_{d}-k x\right)$ and one may solve for $\gamma_{\text {out }}$ and $\varepsilon_{d, \text { out }}^{\prime}$

$$
\begin{gathered}
\tan \gamma_{\text {out }} \cong \frac{\sqrt{\left(1-f^{2} \tan ^{2} \alpha\right)\left(k_{1} m-1\right)\left(1-k_{2} m\right)}}{\cos \alpha(1-k m)} \\
\varepsilon_{d, \text { out }}^{\prime} \cong \varepsilon_{d, \text { out }} \cos \alpha \sqrt{\left(1-f^{2} \tan ^{2} \alpha\right)\left(k_{1} m-1\right)\left(1-k_{2} m\right)}
\end{gathered}
$$

where the new parameter $k_{2}=k \tan (\alpha-\arctan f) / \tan \alpha$ has been introduced, which is generally negative for rubber belts. Then, indicating the boundary point between the two sub-regions with $O(O \equiv D$ if the first sub-region is adhesive), $u_{O}$ can be equated to zero because either $\gamma_{O} \cong 0$ (opening p.) or $\tan \chi_{O}=\rho$ (closing p.) and, integrating Eq. (2) from $\theta_{O}$ to $\theta$ and accounting for Eqs. (1) and (3), one obtains $x^{\prime} \cong \rho+\left[\varepsilon_{d}-\varepsilon_{d, O}+x-x_{O}-\rho\left(\theta-\theta_{O}\right)\right] /$ $\tan \gamma$. Hence, considering that $x_{i n}=\varepsilon_{d, i n} / k_{1}$ and $\varepsilon_{d, O}+x_{O}=\varepsilon_{d, i n}+x_{i n}+\rho\left(\theta_{O}\right.$ $-\theta_{i n}$ ) for both the adhesive and adhesive-like cases and using Eq. (12), one has

$$
x_{\text {out }}^{\prime} \cong \rho+\varepsilon_{d, \text { out }} \cos \alpha(1-k m) \frac{1+m-\frac{\varepsilon_{d, \text { in }}}{\varepsilon_{d, \text { out }}}\left(1+\frac{1}{k_{1}}\right)-\frac{\rho}{\varepsilon_{d, \text { out }}} \Theta}{\sqrt{\left(1-f^{2} \tan ^{2} \alpha\right)\left(k_{1} m-1\right)\left(1-k_{2} m\right)}}
$$

Minding that $x_{\text {out }}^{\prime} / \mathcal{E}_{d, \text { out }}^{\prime}=m$, the division of Eq. (14) by Eq. (13) yields

$$
\begin{aligned}
& m\left(1-f^{2} \tan ^{2} \alpha\right)\left(k_{1} m-1\right)\left(1-k_{2} m\right)= \\
& =\frac{\rho}{\varepsilon_{d, \text { out }} \cos \alpha} \sqrt{\left(1-f^{2} \tan ^{2} \alpha\right)\left(k_{1} m-1\right)\left(1-k_{2} m\right)}+ \\
& \quad+(1-k m)\left[1+m-\frac{\varepsilon_{d, \text { in }}}{\varepsilon_{d, \text { out }}}\left(1+\frac{1}{k_{1}}\right)-\frac{\rho}{\varepsilon_{d, \text { out }}} \Theta\right]
\end{aligned}
$$

which is a sixth degree algebraic equation for $m$, that can be easily solved by a few iterations, in dependence on the drive data, $T_{i n}, T_{\text {out }}$ and $\Theta$.

Expressing the solution for $x$ in the main sliding sub-region by a simple parabolic form $x=x_{\text {out }}+x_{\text {out }}^{\prime}\left(\theta-\theta_{\text {out }}\right)+x_{\text {out }}^{\prime \prime}\left(\theta-\theta_{\text {out }}\right)^{2} / 2$, one may impose the exit conditions $x_{\text {out }}=m \varepsilon_{d, \text { out }}, x_{\text {out }}^{\prime}=m \mathcal{E}_{d, \text { out }}^{\prime}$ and the connection at point $O$ with the upstream adhesive or adhesive-like solution with the same slope $x_{i n}^{\prime}=\rho /\left\{1+0.5 k_{1}[1-\operatorname{sgn}(\rho)]\right\}$. Such continuity conditions yield $\theta_{O}$ 
$=\theta_{\text {out }}-2\left(m \varepsilon_{\text {d,out }}-x_{\text {in }}^{\prime} \Theta-x_{\text {in }}\right) /\left(x_{\text {out }}^{\prime}-x_{\text {in }}^{\prime}\right), x_{\text {out }}^{\prime \prime}=\left(x_{\text {out }}^{\prime}-x_{\text {in }}^{\prime}\right) /\left(\theta_{\text {out }}-\theta_{O}\right)$ and, using the subscript $\ldots . N$ for the drive $N$ pulleys, the axial thrust $F_{z, N} \cong$ $\int_{\text {wrap arc }} S_{t} x d \theta$ turns out to be

$$
\begin{array}{r}
F_{z, N}=\frac{\left(T_{S}-\mu v_{b}^{2}\right) \Theta_{N}}{2 \tan (\alpha+\arctan f)}+\frac{x_{i n, N}^{\prime} S_{t, N} \Theta_{N}^{2}}{2}+ \\
+\frac{2 S_{t, N}}{3 S_{l}} \frac{\left[m\left(T_{T}-\mu v_{b}^{2}\right)-\frac{T_{S}-\mu v_{b}^{2}}{k_{1, N}}-x_{i n, N}^{\prime} \Theta_{N} S_{l}\right]^{2}}{m \frac{\varepsilon_{d, \text { out }}^{\prime}}{\varepsilon_{d, \text { out }}}\left(T_{T}-\mu v_{b}^{2}\right)-x_{i n, N}^{\prime} S_{l}}
\end{array}
$$

where the ratio $\varepsilon_{d, \text { out }}^{\prime} / \varepsilon_{d, \text { out }}$ is given by Eq. (13).

Figures 3 to 6 report the above analytical approximations by dots. Their agreement with the solutions of the full equations is quite acceptable, also in consideration that what is more significant is the whole area under the diagrams and not the local elastic penetration along the arc of contact.

The last equation for completing the formulary is the torque equation. Curtailing the torque values on the driver and driven sides of the torque losses in the bearings if the torque pickups are external to the housing, and averaging them in order take into account the inelastic bending stiffness of the belt, it is possible to write

$$
T_{T}-T_{S}=\frac{1}{2}\left(\frac{M_{R}}{r_{\infty R}}+\frac{M_{N}}{r_{\infty N}}\right)
$$

The given operative data of a V-belt variator are generally the transmitted torque, the speed and the axial thrust on one of the two pulleys, exerted for example by a spring load. According to which axial thrust is given, on the driver or driven side, one has to associate Eq. (17) with either Eq. (11) or Eq. (16) and calculate the unknown belt forces $T_{T}$ and $T_{S}$ on the tight and slack strands. In the case of known driver load, Equation (11) gives the tighter tension directly and then Equation (17) permits calculating the slacker tension. If on the contrary the known axial load is on the driven side, eliminating one of the two tensions, e. g. $T_{S}$, from Eqs. (16-17), one obtains a quadratic equation for the other tension, $T_{T}$ :

$$
a\left(T_{T}-\mu v_{b}^{2}\right)^{2}+b\left(T_{T}-\mu v_{b}^{2}\right)+c=0, \text { where putting } \Delta T=T_{T}-T_{S},
$$




$$
\begin{aligned}
a= & \frac{2 S_{t, N}}{3 S_{l}}\left(m-\frac{1}{k_{1, N}}\right)^{2}+\frac{m \Theta_{N}}{2 \tan (\alpha+\arctan f)} \frac{\varepsilon_{d, \text { out }, N}^{\prime}}{\varepsilon_{d, o u t, N}} \\
b= & \frac{4 S_{t, N}}{3 S_{l}}\left(m-\frac{1}{k_{1, N}}\right)\left(\frac{\Delta T}{k_{1, N}}-x_{i n, N}^{\prime} \Theta_{N} S_{l}\right)+ \\
& +m \frac{\varepsilon_{d, \text { out }, N}^{\prime}}{\varepsilon_{d, \text { out }, N}}\left[\frac{S_{t, N} x_{i n, N}^{\prime} \Theta_{N}^{2}}{2}-\frac{\Theta_{N} \Delta T}{2 \tan (\alpha+\arctan f)}-F_{z, N}\right]-\frac{\Theta_{N} x_{i n, N}^{\prime} S_{l}}{2 \tan (\alpha+\arctan f)} \\
c= & \frac{2 S_{t, N}}{3 S_{l}}\left(\frac{\Delta T}{k_{1, N}}-x_{i n, N}^{\prime} \Theta_{N} S_{l}\right)^{2}-\frac{S_{t, N} S_{l} x_{i n, N}^{\prime 2} \Theta_{N}^{2}}{2}+\frac{\Theta_{N} x_{i n, N}^{\prime} S_{l} \Delta T}{2 \tan (\alpha+\arctan f)}+F_{z, N} x_{i n, N}^{\prime} S_{l}
\end{aligned}
$$

and the driven load can be easily treated as well.

\section{AXIAL THRUST CALCULATION USING THE PROPOSITIONS 24-27} OF ARCHIMEDES' TREATISE " ПEPI EAIK $\Omega N^{\prime}$

It is quite interesting that the axial thrust on the pulley can be alternatively obtained avoiding the integral calculus and using the findings of Archimedes about the areas enclosed by the various branches of a spiral line (see $[5,6])$. These areas were calculated by the Syracusan scientist through very elaborate procedures based on his exhaustion method, as described in the palimpsest of "The Method" (see [7]).

According to the Latin version, the proposition n. 24 states: Spatium comprehensum spirali prima circumactione descripta et linea prima earum, quae in principio circumactionis sunt, tertia pars est circuli primi. Therefore, if $R=a \theta$ is the polar equation of the spiral, $R_{j}=2 \pi j a$ the value of the radius after the $j^{\text {th }}$ revolution and $A_{j}$ the area enclosed by the spiral branch between the points with angular coordinates $\theta=2 \pi(j-1)$ and $\theta=$ $2 \pi j$ and by the segment of the axis $\theta=0$ between the points with radial coordinates $R_{j-1}$ and $R_{j}$, we have $A_{1}=\pi R_{1}{ }^{2} / 3=4 \pi^{3} a^{2} / 3$.

The following proposition n. 25 says: Spatium comprehensum spirali secunda circumactione descripta et linea secunda earum, quae in principio circumactionis sunt, ad circulum secundum eam habet rationem, quam $7: 12, \ldots$ (omissis). In practice, the result is that $A_{2}=7 \pi R_{2}{ }^{2} / 12=$ $28 \pi^{3} a^{2} / 3$ and $A_{2}-A_{1}=6 A_{1}$, as also ascertained in the discussion of proposition 27, where the difference $A_{2}-A_{1}$ is the area enclosed by the first and the second branches of the spiral line and by the segment between the points $R_{1}$ and $R_{2}$ of the axis $\theta=0$.

Furthermore, the proposition 27 states: Spatiorum comprehensorum spiralibus et lineis, quae in circumactione sunt, tertium duplo maius est 
secundo, quartum vero triplo maius, quintum vero quadruplo maius, et semper deinceps insequens spatium toties multiplex erit, quam spatium secundum, quoties indicant numeri ordine sequentes, primum autem spatium sexta pars est secundi. This means in practice that the areas enclosed by two subsequent spiral branches and the axis $\theta=0$ are given by the recursion formula $A_{j}-A_{j-1}=(j-1)\left(A_{2}-A_{1}\right)=8(j-1) \pi^{3} a^{2}$, which result is valid starting from $j=2$.

Summing $A_{1}$ and all the area differences from $j=2$ to $j=n$, it is thus possible to calculate the total area $A_{n}: A_{n}=4 \pi^{3} a^{2}[1 / 3+2(1+2+3+\ldots+$ $n-1]=4 \pi^{3} a^{2}\left(1 / 3+n^{2}-n\right)$. The difference of the $n^{\text {th }}$ circle and $A_{n}$ is a curved triangular stripe, whose area is $\Delta A_{n}=4 \pi^{3} n^{2} a^{2}-4 \pi^{3} a^{2}\left(1 / 3+n^{2}-\right.$ $n)=4 \pi^{3} a^{2}(n-1 / 3)$ and, for very small slope $a$ and very large $n$, as in the V-belt winding, this area is approximately equal to $\Delta A_{n} \cong 4 \pi^{3} a^{2} n$.

Since the radial width of this curved triangular area $\Delta A_{n}$ increases linearly with the distance from its vertex, the area of a segment of angular extension $\Theta$ is $\Delta A_{n \Theta}=\Delta A_{n} \Theta^{2} / 4 \pi^{2} \cong \pi \Theta^{2} a^{2} n=R_{n} \Theta^{2} a / 2$. Considering that $R_{n} \cong r_{\infty}$ and that the spiral slope is $\left.a=r_{\infty} \rho / 1+0.5 \times k_{1}(1-\operatorname{sgn} \rho)\right]$ for the belt-pulley coupling, nearly in the whole arc of contact for driver pulleys and in a large part of it for driven pulleys, we get $\Delta A_{n \Theta} \cong r_{\infty}{ }^{2} \Theta^{2} \times \rho / 12+$ $\left.k_{1}(1-\operatorname{sgn} \rho)\right]$.

Tracing a circle of radius $R_{n} \pm \Delta r$, where $\Delta r$ is the radial penetration at the beginning of the main arc of contact downstream of the small seating region and the positive or negative signs are valid for the closing or opening phases respectively, the absolute value of the radial distance between this circumference and the spiral line gives the local radial penetration, variable along the wrap region.

As the axial push per unit length between the belt and the pulley is obtainable multiplying the radial penetration by the compression-topenetration ratio $2 \tan \alpha$ and by the axial elastic stiffness $E_{z} h / w$ of the belt, the total axial thrust is given by the product of the above curved trapezoidal area, between the spiral and the circumference $R_{n} \pm \Delta r$, and these two quantities:

$$
F_{z}=2 \tan \alpha E_{z} \frac{h}{w}\left[r_{\infty} \Theta \times \Delta r+\frac{r_{\infty}^{2} \Theta^{2} \rho}{2+k_{1}(1-\operatorname{sgn} \rho)}\right]
$$

Minding that $\Delta r=\left(T_{\text {entrance }}-\mu v^{2}\right) r_{\infty} /\left(S_{l} k_{1}\right)$, Equation (19) leads exactly to the same result of Eq. (11), valid for the driver pulleys. Clearly, some discrepancy appears in the case of a driven pulley, where a part of the winding arc is not spiral-shaped. 


\section{CONCLUSIONS}

A very simple model may be derived from the results of a previous analysis of the author on the ratio shift of V-belt variators, whose findings were characterized by a very fine accordance with the experimental results.

The observation of several numerical solutions points out the Archimedean spiral shape of the instantaneous belt path along an extended part of the winding region, with adhesive or adhesive-like conditions for the closing or opening pulleys respectively. In particular, the elastic belt penetration increases in the motion direction for the former and decreases for the latter, independently of the working condition, of driver or driven pulley, that may at most affect the trends of the belt force, of the penetration and of the sliding direction in the following sliding portion of the wrap arc, downstream of the adhesive/adhesive-like sub-region. An easy-to-use formulary has been reported, which may be very useful for design purposes, permitting the evaluation of the axial forces exerted by the pulley walls or else the tension level produced by a given axial thrust on the loaded half-pulley.

It is shown how these calculation may be worked out without recourse to the modern integral calculus, by simply using some propositions of the classical Archimedean treatise On Spirals, as evidence of the up-todateness of Archimedes' thought.

\section{REFERENCES}

[1] Sorge, F., A Theoretical Approach to the Shift Mechanics of Rubber Belt Variators, ASME J. of Mech. Design, 130, 2008, pp. 122602, 1-9.

[2] Cammalleri, M., and Sorge, F., Approximate Closed-Form Solutions for the Ratio Shift Mechanics of Rubber Belt Variators, ASME IDETC2009 Conference, Aug. 30 - Sept. 2, 2009, San Diego, CA, USA.

[3] Archimedes of Syracuse, $\pi \varepsilon \rho i$ ' $\varepsilon \lambda i$ ' $\kappa \omega \nu$ (On Spirals), about 225 B. C. (see [4-7] and Boyer, C.B., A History of Mathematics, Wiley \& Sons, 1968).

[4] Dimarogonas, A.D., Machine Design. A CAD Approach, John Wiley and Sons, Inc., N.Y., 2001.

[5] Heiberg, J.L., Archimedis Opera Omnia, cum Commentariis Eutocii, B.G. Teubner, Leipzig, 1910 1915.

[6] Heath, T.L., The Works of Archimedes, Edited in Modern Notation with Introductory Chapters, Cambridge Univ. Press, 1897.

[7] Heath, T.L., The Method of Archimedes, Recently Discovered by Heiberg. A Supplement to The Works of Archimedes, Cambridge Univ. Press, 1912.

[8] Gerbert, G., Traction Belt Mechanics, Flat belts, V-belts, V-rib belts, Press of the Chalmers Tekniska Högskola, Göteborg, Sweden, 1999. 